\begin{tabular}{c|c|c} 
Eiszeitalter u.Gegenwart & 31 & $23-36$ \\
& & 6 Abb., 1 Tab.
\end{tabular}

\title{
Ein randglaziales Sediment aus der Rißkaltzeit bei Wehr (Südschwarzwald)
}

\author{
HARTMUt Leser *)
}

Riss glaciation, sand, silt, till, parent material, pseudogley, soil profile, granulometrie, major element analysis, depression, Wehra-valley, section, index map.

Southwestern German Massif (Southern Black Forest, Wehr), Baden Württemberg TK 8313

Kurzf a s s ung: Bei der geomorphologischen Kartierung für das Blatt Wehr der GMK 25 wurden im Wehra- und Haseltal Sedimente der Riß-Kaltzeit gefunden. An einem Profil von der Meierhofstraße in Wehr wird der kaltzeitliche und geomorphogenetische Charakter einer sandigen Schluff- und Lehmablagerung diskutiert. Sie erscheint bodentypologisch als Pseudogley. Sedimentologisch handelt es sich um eine randglaziale Ablagerung, in welche Grundmoränenkomponenten der Riß-Kaltzeit eingearbeitet wurden. Als Hypothese wird aufgestellt, daß die Ablagerung des Materials auf einer trogschulterförmigen Verflachung über dem Wehratal-Einschnitt erfolgte.

\section{[A Glacial Border Sediment of the Riss-Glaciation in the Vicinity of Wehr (Southern Black Forest)]}

A bstract: A geomorphological mapping of the sheet Wehr of the GMK 25 (= Geomorphological Map $1: 25000$ ) was carried out. On that occasion sediments of the Riß-Glacial were discovered in the valleys of the river Wehra and the brook Hasel. The author discusses by example of a profile of the Meierhofstraße at Wehr the glacial and geomorphogenetic character of a sandy silt- and loam-deposit. The soil type of the profile is a pseudogley. The sedimentological type of the deposit is characterized as a sediment of the ice-border. In the sediment are worked up ground moraine-components of the Riß-glacial. The hypothesis is discussed that the accumulation of the material took place at a plain with the character of glacial trough shoulders upon the entrenched Wehra-valley.

\section{Einleitung}

Im Rahmen der Erarbeitung von Musterblättern der Geomorphologischen Karte der Bundesrepublik Deutschland $1: 25000$ (= GMK 25) wurde auch das Blatt Wehr kartiert. BARSCH (1976), LESER (1976) und STÄBLEIN (1978) unterrichten über dieses Schwerpunktprogramm der Deutschen Forschungsgemeinschaft $\left.{ }^{1}\right)$. Die Kartierung für die GMK 25 erfolgt im Maßstab $1: 10000$ nach einer standardisierten Baukastenlegende (LESER \& STÄBLEIN 1978). Aufgenommen werden danach geomorphographische und geomorphogenetische Verhältnisse sowie der oberflächennahe Untergrund ${ }^{2}$ ). Vor allem die Darstellung der Informationsschicht „Geomorphogenese“, basierend auf flächendeckender Substratkartierung, Einzelaufschlußuntersuchungen und geomorphographischer Kartierung, erbringt eine Fülle geomorphogenetischer Einzelprobleme. Auch im Bereich des Blattes Wehr wurden solche erkannt und z. T. schon bearbeitet (LESER 1979a, 1980). Diese Einzelprobleme stehen vor

*) Anschrift des Verfassers: Professor Dr. Hartmut L e se r, Geographisches Institut Universität Basel, Klingelbergstraße 16, CH-4056 Basel/Schweiz.

1) Der Deutschen Forschungsgemeinschaft sei auch an dieser Stelle herzlich dafür gedankt, daß die Kartierungsarbeiten sowie die Drucklegung der Karte im Rahmen des GMK-Schwerpunktprogrammes gefördert wurden.

2) Der "oberflächennahe Untergrund“ (auch „Substrat“ genannt) umfaßt Boden, Bodensediment- und Verwitterungsdecken sowie das Gestein, soweit letzteres für die Formbildung bestimmend ist. Keineswegs wird angestrebt, pedologische oder geologische Karten zu ersetzen. Ziel ist vielmehr, den Bezug zwischen "Baumaterial" des Reliefs und Geomorphogenese aufzunehmen und darzustellen. Geomorphologische, geologische und pedologische Karten müssen vom Inhalt her als komplementär begriffen werden. 
allem mit der überregionalen würm- und rißzeitlichen Geomorphogenese im Zusammenhang, die zuletzt HantKe (1978) darstellte. Er stützt sich dabei auf verschiedene Arbeiten von Pfannenstiel und Rahm (besonders 1964), die für das Wehratal die Problematik der pleistozänen Relief- und Sedimentgenese aufarbeiteten.

\section{Problemstellung und Lokalität}

\subsection{Probleme der Rißvereisung im Südschwarzwald}

In der zusammenfassenden Darstellung der rißzeitlichen Landschaftsentwicklung nördlich von Rhein und Aare in Hantke (1978) wird in Anlehnung an die Arbeiten von Pfannenstiel und Rahm (u. a. 1964) für das Wehratal und den Dinkelberg eine ausgedehnte rißzeitliche Vergletscherung angenommen. Diese Verbreitung (Karte 4 bei HANTKE 1978) soll hier nur für einen Bereich des Wehratales diskutiert werden, und zwar

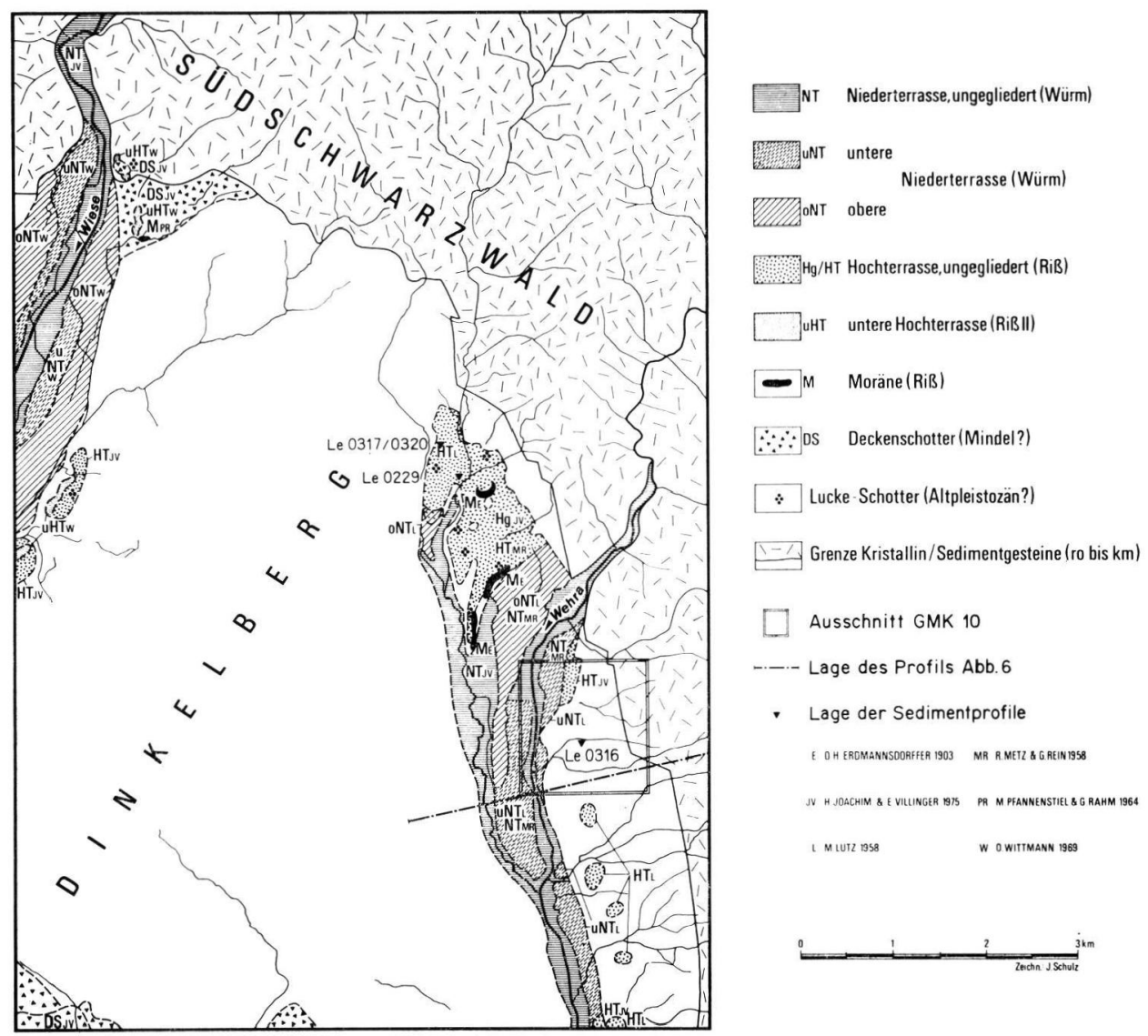

Abb. 1: Die pleistozänen Sedimente des Wehratales nach verschiedenen Autoren und Lage der im Text erwähnten Profile.

Sämtliche Sedimente im Wehratal und auf den Randhöhen werden - mit Ausnahme von ERDMANNSDÖRFER (1903) - durch alle Autoren als Flußterrassensedimente dargestellt. Als Problem ergibt sich, daß in den gleichen Arealen, z. T. aber auch über nachweislich pleistozänen Terrassen, echte Moränensedimente anzutreffen sind. Das beschriebene Profil Le 0316 von der Meierhofstraße in Wehr liegt im Niveau sogenannter Hochterrassenschotter, die inzwischen im Aufschluß als Rißgrundmoräne erkannt werden konnten. 
im Anschluß an die Arbeit Leser (1980). Dort wird die Auffassung von Pfannenstiel \& RAнм (1964) bestätigt, daß zumindest im Wehratal ein rißzeitlicher Gletscher existierte, dessen Grundmoräne Pfannenstiel (1969) von Öflingen beschrieb. Ein ausgedehnteres Vorkommen befindet sich im Raum Hasel (LESER 1980). Von diesen Sedimenten einmal abgesehen konnten bislang keine weiteren, exakt eine Vereisung nachweisenden Ablagerungen gefunden werden. Die Grundmoränen beweisen die Existenz des Gletschers an sich. Uber weitere Einzelheiten der Geomorphogenese des Gebietes ist nichts bekannt. Abbildung 1 stellt die unterschiedlichen Auffassungen über die Sedimente des Wehratales dar, die allein schon bei den Terrasseneinordnungen bestehen. Die von Erdmannsdörffer 1903 auf dem Riedel von Hasel beschriebenen Moränenablagerungen erkannten spätere Autoren nicht an. Durch den Aufschluß von Hasel (Leser 1980) dürften jedoch Zweifel an dieser Auffassung beseitigt sein. Der Aufschluß ist insofern auch interessant, als er sich relativ hoch über dem $\mathrm{Tal}$ befindet und mit den hier zu beschreibenden Ablagerungen halbwegs korrespondiert. Er weist aber, genau wie Pfannenstiel (1969), lediglich den Tatbestand einer Rißvereisung nach. Immerhin wäre wichtig, inwieweit andere Belege die rißzeitliche Gletschergeschichte des Wehratales differenzieren. Als Hypothese wird dabei folgende Überlegung zugrundegelegt: Eine ausgedehnte Vereisung vor dem SchwarzwaldSüdrand, die den gesamten Dinkelberg überzog, ist - trotz der Auffassung von PfanNeNSTIEL \& RAHM (1964) - nicht einwandfrei nachweisbar. Es erhärten sich aber jene Beobachtungen, welche eine differenzierte Eisrandlagenentwicklung um die Ränder des Vorderen Wehratales zwischen dem Austritt aus dem Schwarzwald und dem Eintritt ins Hochrheintal erwarten lassen. Zu diesen Beobachtungen gehört auch das Profil Le 0316.

\subsection{Die Lokalität mit dem Profil Le 0316}

Das Profil befindet sich in $420 \mathrm{~m} \mathrm{NN}$ in der Neubausiedlung an der Meierhofstraße in Wehr (Abb. 2). Die Hochfläche des sogenannten „Kleinen Dinkelberges“ (siehe dazu auch Abb. 6) endet hier. Sie stellt geomorphologisch einen wenig zerriedelten Bergfußhang dar, an den sich östlich die Hänge zur Hotzenwald-Hochfläche und westlich der Abfall zum Wehratal anschließen. Geomorphologische Einzelheiten sind dem Kartenausschnitt der Abbildung $2 \mathrm{zu}$ entnehmen oder der GMK 25 Blatt 4 Wehr (LESER 1979b). Wie ein Blick vom Profilpunkt nach W zeigt, liegt die Hochfläche etwa im Niveau des DinkelbergOstrandes bei Wehr, der verschiedene Tälchen und Abdachungen in Richtung Wehratal aufweist ${ }^{3}$ ). Verglichen mit der Höhenlage der Profile Le 0320 und 0317 in Hasel ergibt sich eine Differenz von $20 \mathrm{~m}$ zu deren $440 \mathrm{~m}$ NN. Die Profile gleichen dem bereits beschriebenen von W Kirche Hasel (= Le 0229; Leser 1980), das mit seiner Oberkante in $405 \mathrm{~m}$ NN liegt. Diese Höhenangaben sind insofern wesentlich, als in sehr unterschiedlichen Höhenlagen um Wehra- und Haseltal offensichtlich rißzeitliche Sedimente vorkommen, die sich z. T. genetisch entsprechen, wie auch die Profile Le 0320, 0317 und 0229. Andererseits muß von der allgemein verbreiteten Vorstellung abgerückt werden, daß genetisch gleiche Sedimente in mehr oder weniger gleichen Höhenlagen über dem Vorfluter vorzukommen haben. Wie bei Gelegenheit an anderer Stelle noch nachzuweisen sein wird, treten zeitlich parallelisierbare und stratigraphisch einwandfreie einzuordnende Terrassensedimente in sehr unterschiedlichen Höhenlagen um das Wehratal auf. Das ist vermutlich auch der Grund dafür, daß sich die in der Literatur beschriebenen Terrassenvorkommen um die Wehra (Abb.1) nur mit Mühen den glazialgeomorphologischen Fakten zuordnen lassen. Vor allem scheint ein unkritisches „Anhängen“ der Terrassenstratigraphie des Wehra-

3) Das damit verbundene geomorphogenetische Problem kann an dieser Stelle nicht behandelt werden. Es bestehen jedoch Beziehungen zwischen diesen Hangformen und der pleistozänen Landschaftsgenese. 
tales an die des Hochrheintales sehr fraglich zu sein (dazu auch Bemerkungen bei LeSER $1979 b)$.

\section{Das Pleistozänprofil von Wehr Meierhofstraße ( = Le 0316)}

Die Aufnahme erfolgte am 20. 10. 1979, nachdem die Lokalität längere Zeit beobachtet worden war. Die Umgebung weist verschiedene Lockersedimentdecken auf, vor allem Lösse und Solifluktionsmaterial (Schutt, umgelagerter Löß, Schotter), die sich in

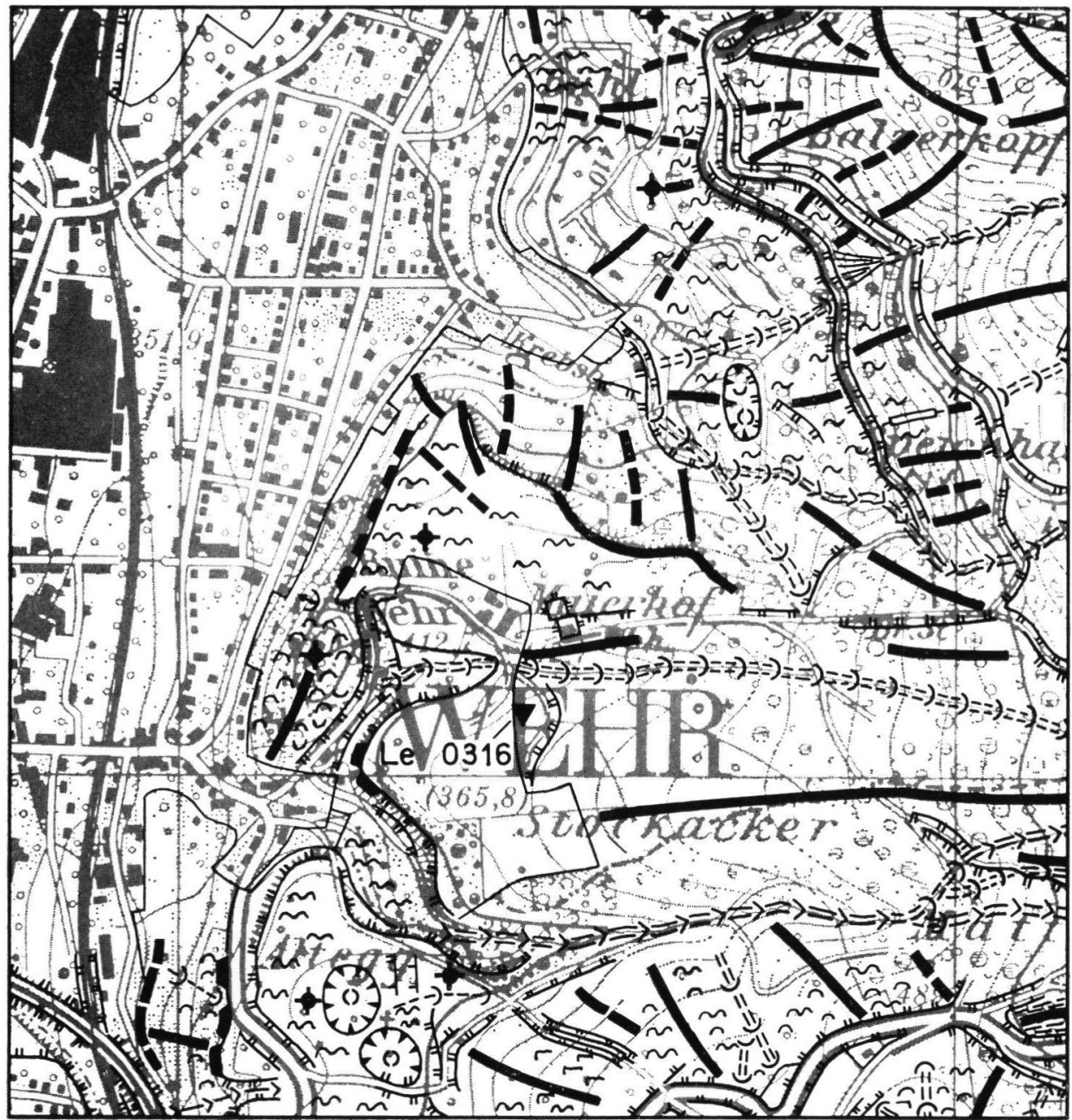

Abb. 2: Ausschnitt aus der Feldkartierung 1:10000 für die GMK 25 Blatt 4 Wehr mit Lage des Profils Le 0316 Meierhofstraße (verkleinert).

Im Neubaugebiet E Wehr wurde das Profil aufgenommen. Die geomorphographische Situation zeigt die Lage auf einer Hangschulter, die sich zum Wehrataleinschnitt leicht absenkt und gegen den Hotzenwald - nach zwischengeschalteten zertalten Riedeln - steil ansteigt. Die Verflachungen oberhalb des Abfalls zum Wehratal lassen sich sedimentologisch nicht als Flußterrassenreste belegen. Um das gesamte Profil Le 0316 herum wurden Erratiker gefunden. - Signaturen, soweit sie sich nicht selbst erklären, siehe Legende der GMK 25 (LESER \& StäbleiN 1978). 
sehr unterschiedlichen Positionen über dem Wehratal befinden und wegen der ungünstigen Aufschlußverhältnisse bisher nicht einzuordnen waren. Bei Bauarbeiten ausgehobene große Erratiker aus Schwarzwaldkristallin ließen jedoch schon früher vermuten, daß auf diesen Randhöhen unmittelbar vor dem Austritt der Wehra aus dem Engtal des Kristallingebietes (Abb. 1) mit glazialen Sedimenten zu rechnen ist. Ohne weiteres wäre, auf Grund der Profile 0320, 0317 und 0229 in Hasel, Grundmoränenmaterial der Riß-Kaltzeit zu erwarten gewesen. Dies entspräche der geomorphologischen und topographischen sowie hydrographischen Situation. Die im Profil 0316 gefundenen Sedimente lassen sich nicht eindeutig als Grundmoränen beschreiben, sondern tragen Merkmale, wie sie bei den würmzeitlichen Eisrandlagensedimenten bei Todtmoos-Au (Leser 1979a) beobachtet wurden. Daß aber Würm als Alter für Profil Le 0316 nicht in Frage kommt, ergibt sich aus verschiedenen, in Abschnitt 4 zu nennenden Gründen.

\subsection{Bodentypologische Beschreibung von Profil Le 0316}

\subsubsection{Profil beschreibung}

Auf dem offensichtlich mehrschichtigen, polygenetischen Sediment hat sich ein Pseudogley entwickelt, dessen relativ starke Ausprägung in einer verhältnismäßig trockenen

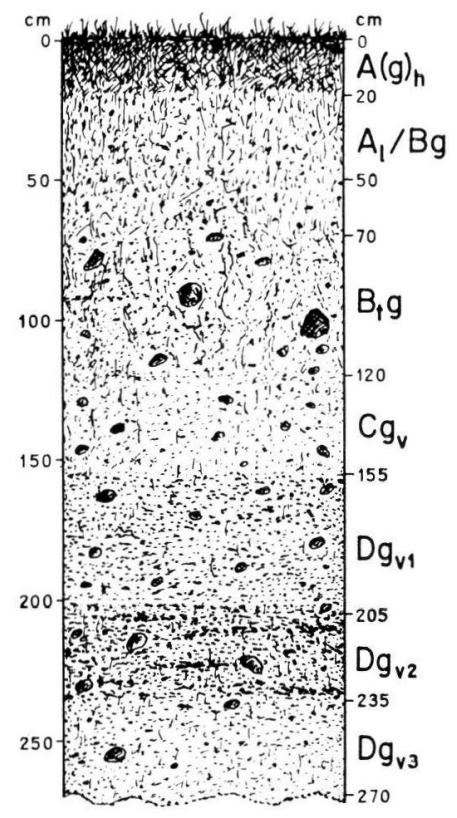

Abb. 3: Darstellung des Profils Le 0316 Wehr Meierhofstraße.

Die Reinzeichnung (H. LESER) basiert auf einer Geländeaufnahme und steht im Zusammenhang mit der Profilbeschreibung in Abschnitt 3.1.1 des Artikels sowie der Darstellung der Analysenergebnisse in Abbildung 4 und Tabelle 1. 
Hochflächenlage für ein hohes Alter spricht. Zur Beschreibung siehe auch Abb. 3 und 4 sowie Tabelle 1.

$20-50 / 70 \mathrm{~cm} \mathrm{Al} / \mathrm{Bg}$

(3) $50 / 70-120 \mathrm{~cm} \mathrm{~B}_{\mathrm{tg}}$

(4)

$120-155 \mathrm{~cm} \mathrm{Cg} v$

(5) $\quad 155-205 \mathrm{~cm} \mathrm{Dg}$ v1

(6) $\quad 205-235 \mathrm{~cm} \mathrm{Dg}$ v2

$235-+270 \mathrm{~cm} \mathrm{Dg}$ v $0-\quad 20 \mathrm{~cm} \mathrm{~A}(\mathrm{~g}) \mathrm{h}$

Blaßbrauner (10 YR 6/3 bzw. 10 YR 4/3)4) sandig-lehmiger Schluff mit Kiesbruch. Sehr dicht und wenig porös. Krümelgefüge. Mäßßig dichte Feindurchwurzelung. Starke Rostfleckung.

Sehr blaßbrauner (10 YR 7/3 bzw. 10 YR 5/4) sandig-lehmiger Schluff. Dicht. Wenig porös. Subpolyedergefüge, leicht plattig. Kiesbruch fein verteilt im gesamten Horizont. Eisen- und Mangankonkretionen durchsetzen gesamte Matrix. Rostfleckung wird zur Horizontuntergrenze (flammig und sehr scharf gegen liegenden Horizont abgesetzt) stärker und dichter, dabei Einzelflecken deutlicher umgrenzt. Einzelne Feinwurzeln im gesamten Profil.

Bräunlich-gelber (10 YR 6/6 bzw. 10 YR 5/8) sandig-schluffiger Lehm. Mit Kiesbruch und einzelnen Steinen um Faustgröße, die z. T. stark verwittert sind (schneidbar). Polyeder- bis Subpolyedergefüge. Horizont marmoriert, z. T. gefleckt und gebändert durch schmale Spalten mit hellgrauen bis weißen Schluff-, Sand- und Kiesfüllungen. Spalten bilden ein unregelmäßiges Muster.

Sehr blaß-brauner (10 YR 7/4 bzw. 10 YR 6/6) sandig-lehmiger Schluffhorizont mit scharfer Ober- und Untergrenze sowie Kiesbruch und einzelnen stark verwitterten Steinen um Faustgröße (wie oben). Material porös, aber dicht bis sehr dicht. Rostflecken. Eisen- und Mangankonkretionen durchsetzen gesamte Matrix (1 bis $2 \mathrm{~cm}$ Durchmesser). Gefüge nicht erkennbar, Material jedoch kohärent.

Sehr blaß-brauner (10 YR 7/4 bzw. 10 YR 5/6) bis gelblich-brauner sandiger Lehm mit scharfer Obergrenze, jedoch Übergangssaum zum liegenden Horizont. Kies und Grobsand. Steine (stark verwittert, ca. 4-10 cm L5) ) im gesamten Horizont, ebenso Rostflecken. Bändchenstruktur im gesamten Horizont, jedoch schwach ausgebildet (schichtungsähnlich). Gefüge nicht erkennbar, Material kohärent.

Rötlich-gelber (7.5 YR 6/6 bzw. 7.5 YR 5/6) bis intensiv-brauner sandig-schluffiger Lehm mit Farbunterschied zum liegenden und hangenden Horizont. Sonstige Merkmale: Wie $\mathrm{Dg}_{\mathrm{v} 1}$.
Sehr blaß-brauner (10 YR 7/4 bzw. 10 YR 6/6) schluffig-lehmiger Sand, dessen Basis nicht aufgeschlossen ist, mit Farbunterschied zum hangenden Horizont. Sonstige Merkmale: Wie $\mathrm{Dg}_{\mathrm{v} 1}$.

\subsubsection{Pedogenetisch-sedimentologische Deutung des Profils}

Bodenfarbe sowie verschiedene chemische und physikalische Merkmale (siehe dazu Abb. 4 und Tab. 1) weisen den Boden als einen Pseudogley aus, der typologisch zwischen primärem und sekundärem Pseudogley (Schaffer \& Schachtschabel 1976: 340 ff) steht. Überwiegen morphologisch Merkmale des primären Pseudogleys, weisen die Nährstoffarmut sowie Konkretionen und Rostfleckenausbildung auf einen sekundären hin. Die bodentypologische Einordnung ist jedoch für die geomorphologische Problemstellung nur von zweitrangiger Bedeutung. Die starke Verwitterung des Bodens weist aber auf ein hohes Alter hin. Für die Genese wesentlich scheint die sedimentologische Grenze zwischen dem Profiloberteil $\left(\mathrm{A}(\mathrm{g})_{\mathrm{h}}\right.$ bis $\left.\mathrm{Cg}_{\mathrm{v}}\right)$ und dem Unterteil $\left(\mathrm{Dg}_{\mathrm{v} 1}\right.$ bis $\left.\mathrm{Dg}_{\mathrm{v} 3}\right)$, die sich im gröbe-

4) Farben nach Munsell Soil Color Charts, Baltimore 1954. Zuerst wird der Trockenwert (= labortrocken), danach der Feuchtwert (= feldfrisch) angegeben.

5) $\mathrm{L}=$ Maximale Länge (Längste Achse in der Ebene der Abplattung). 

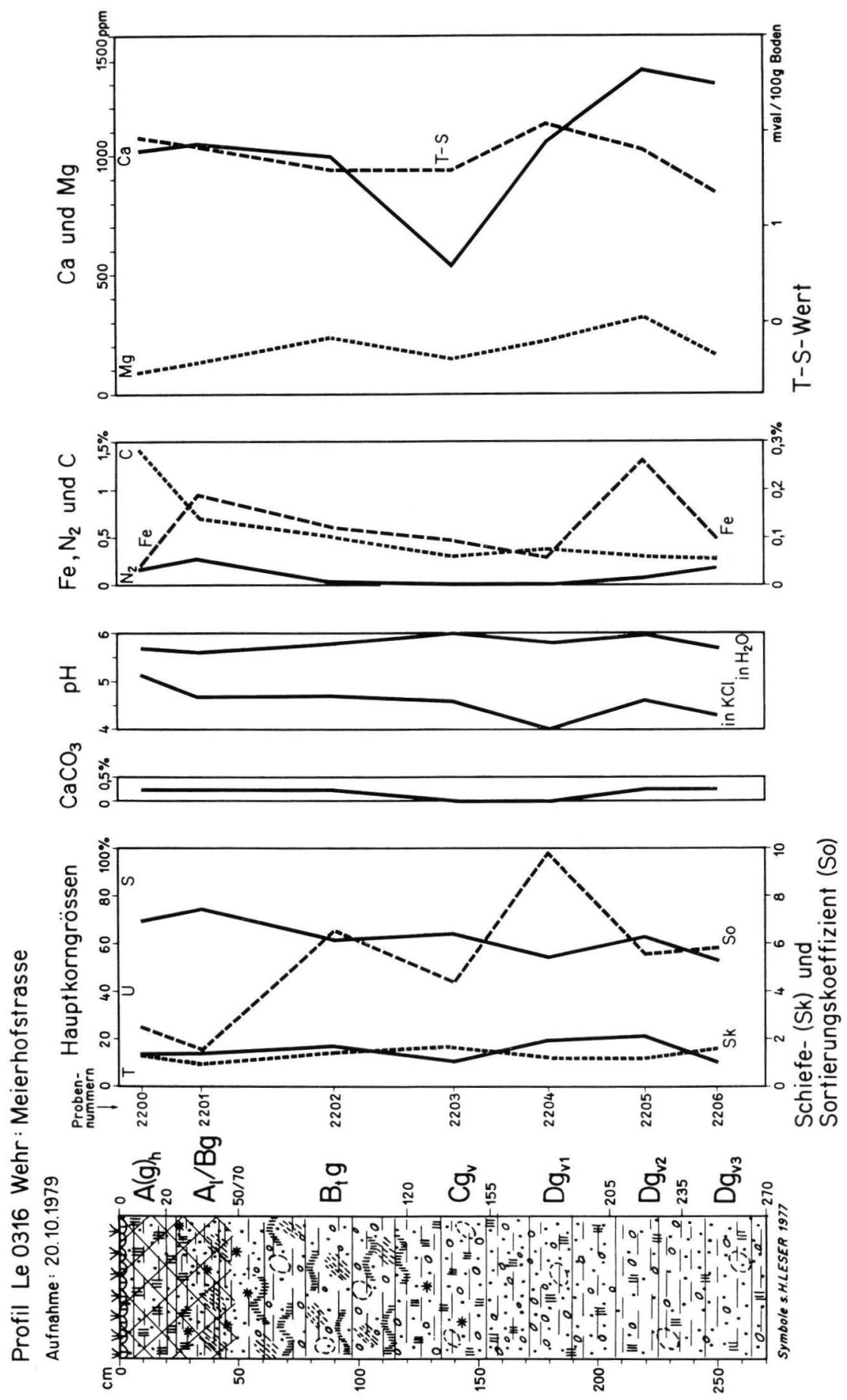

Abb. 4: Auszugsweise Darstellung der Analysendaten von Profil Le 0316.

Analysiert wurden die Proben Le 2200 bis 2206. Entnahme erfolgte in der Horizont- bzw. Schichtmitte. Analysenverfahren nach Schlichting \& Blume „Bodenkundliches Praktikum“ (1966) sowie LESER "Geomorphologische Feld- und Labormethoden“ (1977). 


\begin{tabular}{|c|c|c|c|c|c|c|c|c|c|c|c|c|c|c|c|c|c|c|c|c|}
\hline \multirow{3}{*}{$\begin{array}{l}\text { Probe } \\
\text {-Nr. }\end{array}$} & \multirow{3}{*}{ Material } & \multirow{3}{*}{$\begin{array}{c}\text { Entnahme } \\
\text { tiefe in } \\
\mathrm{cm}\end{array}$} & \multirow{3}{*}{$\begin{array}{l}\text { Gew \% } \\
<2 \mathrm{~mm}\end{array}$} & \multicolumn{12}{|c|}{ Korngrößenklassen des Feinsediments $2.000 \mathrm{~mm}$} & \multirow{2}{*}{\multicolumn{2}{|c|}{ Koeffizienten }} & \multirow{2}{*}{\multicolumn{3}{|c|}{ Quartilsmaße in mm }} \\
\hline & & & & 2.000 & 1.000 & 0.500 & 0.250 & 0.125 & 0.063 & 0.032 & 0.016 & 0.008 & 0.004 & 0.002 & $<0,001$ & & & & & \\
\hline & & & & 1.000 & 0.500 & 0.250 & 0.125 & 0.063 & 0.032 & 0.016 & 0.008 & 0.004 & 0.002 & 0.001 & & Sk & So & $a_{1}$ & $a_{2}$ & $o_{3}$ \\
\hline 2200 & $\begin{array}{l}A(g) h \text { eines } \\
\text { Pseudogleys }\end{array}$ & 10 & 100.00 & 7.1 & 7.0 & 6.9 & 4.9 & 4.4 & 23.3 & 18.3 & 11.5 & 0.0 & 2.2 & 14.4 & 0.0 & 1.24 & 3.31 & 13.0 & 34.8 & 142.0 \\
\hline 2201 & $\begin{array}{l}A_{I} / B g \text { eines } \\
\text { Pseudogleys }\end{array}$ & 35 & 100.00 & 6.3 & 5.6 & 5.9 & 3.8 & 3.0 & 48.7 & 6.1 & 0.1 & 1.8 & 4.7 & 14.2 & 0.0 & 0.92 & 1.56 & 25.8 & 43.6 & 62.2 \\
\hline 2202 & $\begin{array}{l}\mathrm{B}_{\mathrm{t} g} \text { geines } \\
\text { Pseudogleys }\end{array}$ & 90 & 100.00 & 10.6 & 9.5 & 8.3 & 5.2 & 4.6 & 15.0 & 0.5 & 21.5 & 0.9 & 7.2 & 16.7 & 0.0 & 1.42 & 6.50 & 7.9 & 36.3 & 332.1 \\
\hline 2203 & $\begin{array}{l}\mathrm{Cg} g_{\mathrm{v}} \text { eines } \\
\text { Pseudogleys }\end{array}$ & 140 & 100.00 & 9.8 & 8.7 & 7.3 & 5.3 & | 4.4 & 21.9 & 15.8 & 9.6 & 2.7 & 3.9 & 10.6 & 0.0 & 1.55 & 4.44 & 13.8 & 39.5 & 269.8 \\
\hline 2204 & $\begin{array}{l}\mathrm{Dg}_{\mathrm{g}_{1}} \text { eines } \\
\mathrm{P}_{\text {seudogleys }}\end{array}$ & 180 & 100.00 & 12.5 & 12.1 & 9.5 & 6.2 & 5.0 & 8.7 & 15.2 & 4.0 & 2.9 & 4.1 & 19.8 & 0.0 & 1.16 & 9.78 & 5.1 & 43.0 & 485.7 \\
\hline 2205 & $\begin{array}{l}\mathrm{Dg} \mathrm{g}_{\mathrm{v} 2} \text { eines } \\
\text { Pseudogleys }\end{array}$ & 220 & 100.00 & 10.8 & 8.7 & 7.1 & 5.7 & 4.6 & 23.3 & 9.8 & 6.6 & 0.7 & 1.8 & 20.9 & 0.0 & 1.23 & 5.63 & 9.3 & 42.4 & 292.3 \\
\hline 2206 & $\begin{array}{l}\mathrm{Dg}_{\mathrm{v} 3} \text { eines } \\
P_{\text {seudogleys }}\end{array}$ & 250 & 100.00 & 14.1 & 11.0 & 9.2 & 6.6 & 5.2 & 17.0 & 11.5 & 8.7 & 1.1 & 5.4 & 10.2 & 0.0 & 1.64 & 5.77 & 15.2 & 53.4 & 503.2 \\
\hline
\end{tabular}

Tab. 1: Analysenergebnisse der Proben Le 2200-2206 aus dem Profil Le 0316 Wehr Meierhofstraße.

ren Korn der liegenden Horizonte $(155-+270 \mathrm{~cm}$ unter Flur), aber z. T. auch in deren Chemismus ausdrückt. Insgesamt dürfen jedoch die Unterschiede - vor allem der physikaiischen Eigenschaften - zwischen Ober- und Unterteil des Profils nicht überbewertet werden. Darauf weist nämlich der Steingehalt aller Horizonte unterhalb 50/70 cm unter Flur hin. Bei diesen Steinen (Abb. 5) handelt es sich um stark verwitterten Gneis aus dem Schwarzwald. Sie haben plattige Gestalt, sind stark kantengerundet bis kantig, bei Dikken von $4-6 \mathrm{~cm}$ und Längen von um $10 \mathrm{~cm}$. Ihre starke Verwitterung zeigt sich an einer

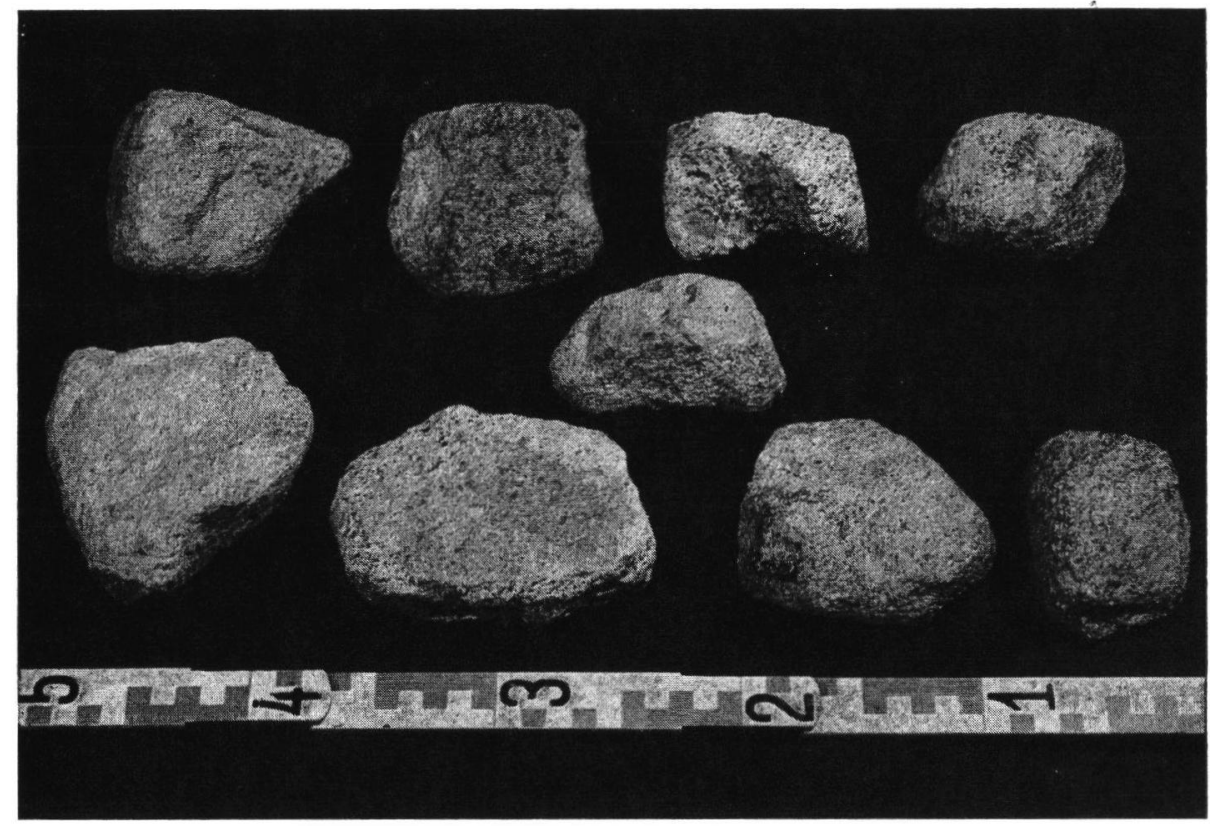

Abb. 5: Handstücke der Probe Le 2207 aus dem Profil Le 0316 Wehr Meierhofstraße.

Die Komponenten sind auch im Bild als stark verwittert zu erkennen. Trotzdem ist die moränale Formgestalt noch gut sichtbar. Die starke Beanspruchung der Steine geht auf die Aufarbeitungsprozesse im Zuge der Sedimentablagerung und der Gebietsgeomorphogenese zurück, für die ein Eisrückzug auf den Randhöhen oberhalb des Wehra-Taleinschnittes angenommen wird. 


\begin{tabular}{|c|c|c|c|c|c|c|c|c|c|c|c|c|c|c|c|}
\hline \multicolumn{2}{|c|}{ Farbe } & \multicolumn{2}{|c|}{$\mathrm{pH}$} & \multirow{2}{*}{$\begin{array}{c}\text { Kalk-u.Do- } \\
\text { lomitgehalt } \\
\% \mathrm{CaCO}_{3}\end{array}$} & \multirow{2}{*}{$\% \mathrm{~N}_{2}$} & \multirow{2}{*}{$\% \mathrm{Fe}$} & \multirow{2}{*}{$\% \mathrm{C}$} & \multirow{2}{*}{$\begin{array}{l}\% \text { org. } \\
\text { Mat. }\end{array}$} & \multirow{2}{*}{$\begin{array}{l}\text { Phosphat } \\
\text { (ppm) }\end{array}$} & \multirow{2}{*}{$\begin{array}{c}\mathrm{Ca} \\
\text { (ppm) }\end{array}$} & \multirow{2}{*}{$\begin{array}{c}\mathrm{Mg} \\
(\mathrm{ppm})\end{array}$} & \multirow{2}{*}{$\begin{array}{c}-\mathrm{K} \\
(\mathrm{ppm})\end{array}$} & \multirow{2}{*}{$\begin{array}{c}\mathrm{S} \\
\text { (m val pro } \\
100 \mathrm{~g} \text { Boden) }\end{array}$} & \multirow{2}{*}{$\begin{array}{c}\mathrm{T} \\
(\mathrm{m} \text { val pro } \\
100 \mathrm{~g} \text { Boden })\end{array}$} & \multirow{2}{*}{$\begin{array}{l}V \\
(\%)\end{array}$} \\
\hline feucht & trocken & in $\mathrm{H}_{2} \mathrm{O}$ & in $\mathrm{KCl}$ & & & & & & & & & & & & \\
\hline $\begin{array}{c}10 Y R \\
4 / 3\end{array}$ & $\begin{array}{c}10 Y R \\
6 / 3\end{array}$ & 5.70 & 5.16 & 0.236 & 0.168 & 0.04 & 1.399 & 2.49 & 16 & 1020 & 93 & 20 & 58.0 & 59.92 & 96.79 \\
\hline $\begin{array}{c}10 Y R \\
5_{/ 4}\end{array}$ & $\begin{array}{c}10 Y R \\
7 / 3\end{array}$ & 5.55 & 4.65 & 0.235 & 0.274 & 0.19 & 0.699 & 1.244 & 52 & 1050 & 117 & 23 & 59.0 & 60.84 & 96.97 \\
\hline $\begin{array}{c}10 \mathrm{YR} \\
5_{/ 8}\end{array}$ & $\begin{array}{c}10 Y R \\
6 / 6\end{array}$ & 5.82 & 4.68 & 0.234 & 0.046 & 0.12 & 0.502 & 0.893 & 2 & 1000 & 238 & 58 & 59.4 & 61.0 & 97.37 \\
\hline $\begin{array}{c}10 \mathrm{YR} \\
6 / 6\end{array}$ & $\begin{array}{c}10 Y \mathrm{YR} \\
7 / 4\end{array}$ & 5.96 & 4.55 & 0 & 0.008 & 0.095 & 0.299 & 0.532 & 5 & 540 & 145 & 28 & 61.0 & 62.6 & 97.44 \\
\hline $\begin{array}{c}10 \mathrm{YR} \\
5 / 6\end{array}$ & $\begin{array}{c}10 Y R \\
7 / 4\end{array}$ & 5.80 & 4.01 & 0 & 0.008 & 0.063 & 0.366 & 0.651 & 4.5 & 1060 & 237 & 78 & 50.4 & 52.48 & 96.03 \\
\hline $\begin{array}{c}7.5 \mathrm{YR} \\
5 / 6\end{array}$ & $\begin{array}{c}7.5 Y \mathrm{R} \\
6 / 6\end{array}$ & 5.97 & 4.58 & 0.234 & 0.062 & 0.266 & 0.299 & 0.532 & 4.5 & 1360 & 322 & 70 & 60.8 & 62.64 & 97.06 \\
\hline $\begin{array}{c}10 Y R \\
6 / 6\end{array}$ & $\begin{array}{c}10 Y R \\
7 / 4\end{array}$ & 5.74 & 4.29 & 0.237 & 0.156 & 0.102 & 0.268 & 0.477 & 4 & 1200 & 167 & 42 & 58.8 & 60.16 & 97.37 \\
\hline
\end{tabular}

Art Rindenbildung („Rostkrusten“ von 0,5 cm Dicke), Angrusung, Feinrissen verschiedener Generationen sowie Abschuppungs- und Abschalungseffekten. Andeutungen von Schlagmarken kommen ebenfalls vor. Auffällig ist auch die „moränale Form“, ohne daß den stark verwitterten Stücken mit absoluter Sicherheit Geschiebecharakter zukommt. Daneben treten noch solche Stücke auf, die in der Wand vergrusten und daher nicht entnommen werden konnten. Auf starke Verwitterung weisen auch die Grobsand- und Kiesbändchen hin, welche die Aufschlußwand stellenweise schwach bändern. Die Schichtung ist leicht gewellt. Kies und Grobsand weisen Eisen- und Manganhüllen und -verhärtungen auf. Beim Abtrocknen der Wand beginnen die Bändchen aufzublättern. Diese Sedimentstruktur ist am deutlichsten in den Horizonten $\operatorname{Dg}_{\mathrm{v} 1}$ bis $\mathrm{Dg}_{\mathrm{v} 3}$. Die große äußerliche Ahnlichkeit aller Horizonte wird vor allem durch die intensive Verwitterung des Gesamtprofils - letzthin durch die Bodenbildung - bewirkt. Dies war auch der Grund, den Profilunterteil-Horizonten das Symbol „v“ beizugeben. - Aus diesen Fakten kann geschlossen werden, daß allein schon wegen des gleichmäßigen Steingehaltes aller Horizonte kein Hiatus im Profil vorliegt, auch nicht zwischen $\mathrm{Cg}_{\mathrm{v}}$ und $\mathrm{Dg}_{\mathrm{v}} \mathbf{1}$. Es handelt sich vielmehr um normale sedimentologische Differenzierungen, wie sie für einen länger dauernden geomorphologischen Prozeß charakteristisch sind.

\section{Zur Profil- und Gebietsgeomorphogenese}

\subsection{Der kaltzeitliche Charakter der Sedimente}

Auszugehen ist vom Material. Die vorherrschende Schluffkorngröße in mehreren Horizonten darf nicht darüber hinwegtäuschen, daß es sich beim Material nicht um Löß handelt. Das schließt nicht aus, daß Löß in das Sediment eingearbeitet wurde. Gegen den Lößcharakter sprechen:

(1) Das Fehlen von Primärlößmerkmalen.

(2) Der hohe Kiesanteil in allen Horizonten.

(3) Das gleichmäßige Vorkommen von Steinen in praktisch allen Horizonten.

(4) Die partiell deutliche aquatisch bedingte Schichtigkeit.

In jedem Fall muß aber das Sediment als kaltzeitlich entstanden angesprochen werden. Dafür gibt es zahlreiche Kriterien: 
(1) Frostspalten pseudomorphosen (Kryotexturformen): Sie treten bis um $100 \mathrm{~cm}$ unter Flur auf, was der Tiefe der Auftauschicht des pleistozän-periglazialen Permafrostgebietes entspricht. Diese besonders im $B_{t}$ g-Horizont auftretenden Frosttrockenrisse wurden unter noch kaltzeitlichen Verhältnissen wieder mit Feinsedimenten verfüllt. Da ihr Auftreten auch auf die Würmkaltzeit zurückgehen könnte, muß ihr genetisch-chronologischer Aussagewert zunächst etwas relativiert werden.

(2) Eisen- und Manganbänderungen: Sie lehnen sich an ablagerungsbedingte Sedimentunterschiede an (Sandbändchen, Kieslagen). Sie gelten als typisch für die klimatisch bedingten Wechsel im Zustand des Permafrostbodens.

(3) Schichtstörungen: Die nicht sehr ausgeprägten und nicht überall im Profil durchverfolgbaren Schichten weisen deutlich erkennbare Lagerungsstörungen in Form von leichten Schichtverbiegungen auf, die nur durch post- und (vielleicht auch) synsedimentäre Taueffekte erklärbar sind, die beim Niedertauen von kleineren oder größeren Toteislinsen auftreten (auch im Sinne des Beispiels von SHaw \& ArCHER 1979: 354).

(4) $\mathrm{K}$ i esbruch : Er ist in faktisch allen Horizonten anzutreffen und spricht für die kaltzeitlichen Verwitterungs- und Transportprozesse unter periglazialen Bedingungen.

(5) A ufarbeitung von Löß: Wegen der Lößvorkommen in der näheren und weiteren Umgebung (Ablagerung z. T. in großen Taschen im liegenden Sediment) muß davon ausgegangen werden, daß ein Teil der Schluffkomponente im Sediment auf umgelagerten und aufgearbeiteten Löß zurückgeht. Solche Aufarbeitungen sind für Periglazialgebiete typisch.

(6) Aufarbeitung von Geschieben: Die o. a. stark verwitterten Gesteinsstücke lassen sich von der Form her mit stratigraphisch eindeutigem Moräneschutt rißzeitlicher Schwarzwaldsedimente vergleichen (siehe Abb. 5 in dieser Arbeit und Abb. 6 in Leser 1979a sowie Abb. 5 in LeSER 1980). Die starke Verwitterung hat diese Form etwas beeinträchtigt, ebenso auch ein glazifluvialer Transport. Wegen beider Kriterien sowie durch das Vorkommen zusammen mit Frostbodenerscheinungen wird der kaltzeitliche Charakter des Sediments zusätzlich erhärtet.

(7) Frostgesprengte Gerölle und Gesteinsbruchstücke: Sie kommen in allen Horizonten vor, vor allem aber unter dem eigentlichen Boden. Die Frostsprengung erfolgte syngenetisch, d. h. sie trat während oder unmittelbar nach der Sedimentation auf, so daß die schmalen Zwischenräume zwischen den Bruchstellen mit Sediment verfüllt werden konnten.

(8) Erratiker: Beim Ausheben von Baugruben unmittelbar neben dem Aufschluß wurden Kristallinblöcke gefunden, die eindeutig als Erratiker anzusprechen sind, die Pfannenstiel \& Rahm (1964) für weite Bereiche des Wehra- und Wiese-Vereisungsgebietes nachweisen. Für den Glazialcharakter der Blöcke sprechen die Sichelmarken auf den Oberflächen (SchwarzBACH 1978) ${ }^{6}$ ).

\subsection{Das rißzeitliche Alter der Sedimente}

Aus der Gesamtlage des Profils im Wehragebiet und zu den bekannten Glazial- und Periglazialsedimentvorkommen ist mit Sicherheit rißzeitliches Alter für den Aufschluß anzunehmen. Die Ablagerung ist zwar kein Moränensediment, wie aber noch zu zeigen sein wird, ein solches aus dem Eisrandlagenbereich. Nach den Vorstellungen von Pfannenstiel

6) Trotz bisher zahlreicher ausgegrabener Erratiker auf den Randhöhen um das Wehratal konnte der Verfasser noch keinen aus einem Aufschluß dokumentieren. Eine Einzelbeobachtung in einem Aufschluß am Ortsrand von Offlingen (TK 258413 Säckingen) war durch plötzliche Bauarbeitenfortschritte nicht mehr bearbeitbar. 
\& Rahm (1964) sowie HantKe (1978) gilt eine Rißvereisung für das Wehragebiet als sicher. Die Lokalität liegt absolut innerhalb des angenommenen Vereisungsbereiches. - Als Einzelkriterien für rißzeitliches Alter wären anzuführen:

(1) Höhen lage der Sedimente : Das Profil Le 0316 liegt zwar nicht in absolut gleicher Höhe wie die bisher nachgewiesenen Rißgrundmoränen von Öflingen (PFANNENSTIEL 1963) und Hasel (LESER 1980), was auch nicht entscheidend zu sein braucht. Die Höhendifferenz der Meierhofstraße in Wehr mit dem Profil zur Grundmoräne von Hasel beträgt nur $+15 \mathrm{~m}$ und zur Stützergrundstraße in Hasel nur $-20 \mathrm{~m}$. Diese Beträge bewegen sich in Größenordnungen, die für die Gletschertätigkeit auch in einem Mittelgebirge immer noch als normal anzusehen sind. Entscheidender ist die Höhenlage zu den würmzeitlichen Ablagerungen, die sich alle weit unterhalb dieser Niveaus, im Bereich des engeren Wehratales, befinden. Würmzeitliches Alter bleibt also aus Gründen der stratigraphischen Position völlig ausgeschlossen. $\mathrm{Da}$ ein anderes Erdzeitalter für ein glazial-periglaziales Sediment mit den o. a. Eigenschaften (Abschnitt 4.1.) in dieser Position und in diesem Raum nicht in Frage kommt, bleibt — nach Ausschluß von Würm - nur Riß übrig. (2) Erratiker : Die bereits erwähnten Kristallinblöcke weisen in dieser topographischen Lage auf eine Überdeckung mit Rißeis hin.

(3) Ges chiebe : Die moränale Form der Steine im Profil deutet auf zunächst vorhergehende Ablagerungen von (Grund-)Moräne (s. auch Abschnitt 4.1. (6)). Die Aufnahme des Materials und Aufarbeitung ist nur möglich, wenn vorher Eis über das Gebiet hinwegging. Eine Vereisung des Sedimentationsgebietes oberhalb des Wehratales war aber nur während des Riß möglich.

\subsection{Die geomorphogenetische Interpretation der Sedimente}

Zunächst soll ausgeschlossen werden, was geomorphogenetisch in dieser Position des Profils Le 0316 zumindest denkbar wäre, obwohl sich durch die o. a. Kriterien und Merkmale des Sedimentes die geomorphogenetische Interpretation ziemlich stark auf eine Lösung verengt. Nicht in Frage kommt die Deutung als:

(1) Ha ngfußsedime n t: Dagegen sprechen, trotz des „Hinterlandes“, die Schichtung und die Erratiker, sowie der Sedimentcharakter.

(2) A morphes Kryosolifluktionssediment: Dagegen spricht die Schichtung der Ablagerungen sowie das Fehlen ausgesprochenen Solifluktionsschuttes.

(3) Periglaziales Schwemmsediment: Dagegen sprechen das Auskeilen der Bändchen und Schichten über die gesamte Profilwand hinweg, das Fehlen einer Basisdiskordanz sowie die insgesamt zu schwache Schichtprägung.

Nach Ausschluß dieser Möglichkeiten bleibt als Lösungsmöglichkeit offen: Es handelt sich um ein randglaziales Sediment, vielleicht vom Charakter einer "Schwemm-Moräne“ (flow till), die vor einer Eisrandlage im Zuge des zurückweichenden Eises abgelagert wurde. Wie die sedimentologischen Hinweise auf Niedertaueffekte zeigen, muß Toteisvorkommen angenommen werden. Hier wird auch das Konzept des „waterlain till“ (Dreimanis 1979) tangiert, der bekanntlich auch genetische Beziehungen zu glazifluvialen Ablagerungsbedingungen aufweist. - $\mathrm{Zu}$ diesen Deutungen paßt, daß mehrere Kriterien einwandfrei für Eisrückzug sprechen:

(1) Lage des Sediments: Eisrandlagensedimente des Riß sind für den Rückzugsweg wahrscheinlich, weil ein Vorstoß des Rißeises des Wehragletschers mindestens bis Óflingen (ca. 3,5 km S von Wehr Meierhofstraße) nachgewiesen ist.

(2) Ni ederta u effekte: Sie treten entweder im Gletschereisrandbereich oder über Toteislagen und -linsen vor dem noch geschlosseneren Gletscherzungenende auf. 
(3) A ufgearbeitete Geschiebe: Die Geschiebe und/oder glazifluvialen Schotter im Sediment gehören offensichtlich einer Vorrückungsphase an (Grund- oder Endmoränenrelikte sowie Vorrückschotter). Das Material wurde sehr gründlich aufgearbeitet, was sich an der intensiven Verwitterung der Komponenten (die durch Transport sicher vorbereitet wurde) sowie an der Verteilung im Sedimentkörper des Profils ablesen läßt.

\section{Hypothese zur Rißvereisung im Wehratal}

Unbestritten ist die Rißvereisung des Wehratales schon lange, wenngleich über die genaue Eisverbreitung und die Dynamik des Eisvorstoßes und -rückzuges kaum etwas bekannt war. Die bisherigen Grundmoränenvorkommen liegen alle im Bereich des engeren Wehratales. Daher wurde schon darauf hingewiesen, (LEser 1979 b, 1980), daß eine Eisüberdeckung des gesamten Dinkelberges, wie sie andere Autoren postulierten, erst noch flächendeckend zu belegen wäre. Für eine differenzierte Eisranddynamik sprechen nun das Profil Le 0316, sowie die Vorkommen der Grundmoränen von Oflingen und Hasel. Wird

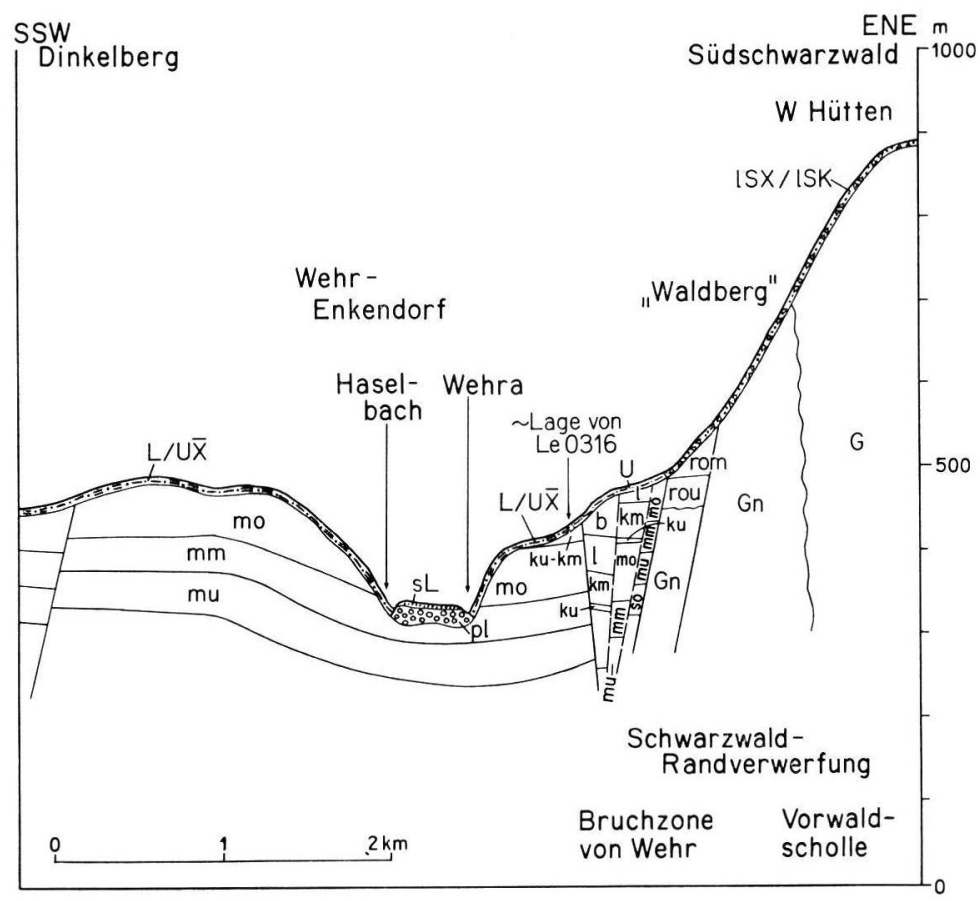

Abb. 6: Geologisch-geomorphologisches Profil durch das Wehratal wenig S Wehr.

Das Profil wurde der Arbeit Joachim \& Villinger (1975) entnommen und durch sedimentologische Angaben aus der GMK-Aufnahme verändert. Erkennbar ist die trogschulterähnliche Form der Randhöhenflächen auf der Hotzenwald-Seite des Wehratales, wo sich auch das Profil Le 0316 befindet. Beachtenswert ist die offensichtliche Kappung der mesozoischen Schichten. Es handelt sich bei den Hangverflachungen demnach um Skulpturformen.

Geologische Zeichenabkürzungen: $\mathrm{G}=$ Granit; $\mathrm{Gn}=\mathrm{Gneis}$; rou/rom = Unteres und Mittleres Rotliegendes; so $=$ Oberer Buntsandstein; $\mathrm{mu} / \mathrm{mm} / \mathrm{mo}=$ Unterer, Mittlerer und Oberer Muschel$\mathrm{kalk} ; \mathrm{ku} / \mathrm{km}=$ Unterer und Mittlerer Keuper; $1=$ Lias; $\mathrm{b}=$ Dogger; $\mathrm{pl}=$ Pleistozäne Flußterrassenschotter. Symbole des oberflächennahen Untergrundes $(0-1 \mathrm{~m}): \mathrm{U}=$ Schluff; $s \mathrm{~L}=$ sandiger Lehm; L/UX $=$ Lehm mit Schluff und beigemischten plattigem Kalkschutt; 1SX/1SK = lehmiger Sand mit kantigem Schutt unter und über $200 \mathrm{~mm}$ Kantenlänge. 
vor dem Hintergrund der hier nur grob skizzierten Eisverbreitung das Querprofil des Wehratales auf der Höhe von Wehr-Enkendorf interpretiert (Abb. 6), ist folgende Schlußfolgerung möglich: Zumindest andeutungsweise läßt sich das Talquerprofil als glazial geformt beschreiben. So nimmt HANTKE (1978) in Anlehnung an verschiedene andere Autoren eine Flanke des Rißeises auf den Randhöhen des Wehratales unterhalb des Hotzenwaldabfalls an (dort Karte 4), d. h. etwa im hier beschriebenen Arbeitsgebiet. Die Talgestalt weist in der Tat eine Art Trogschultern auf — ohne daß damit das Wehratal als Trogtal bezeichnet werden soll. Die Eisausbreitung über das engere Tal hinaus dürfte sich jedoch ähnlich der auf Trogschultern vorzustellen sein. Der Abbildung 6 wurde bewußt nicht eine selbst erarbeitete Profillinie zugrunde gelegt, sondern eine aus der hydrogeologischen Arbeit von JoAchim \& VILlinger (1975), der auch die Gesteinslagerung (nicht jedoch die Lockersedimentverbreitung) entnommen wurde.

Für die Hypothese sprechen weitere Beobachtungstatsachen:

(1) Das Querprofil kann mehr oder weniger ähnlich oder gleich über das gesamte Vordere Wehratal hinweg verfolgt werden. Im Kristallin ändert sich das Profil natürlich. Das geht einerseits auf die dort stärkeren tektonischen Hebungen während des Pleistozäns zurück (RAнм 1961), andererseits auf den plötzlichen Wechsel der Gesteinsverhältnisse (Mesozoikum zum Kristallin). Allerdings können auch hoch über dem Wehra-Engtal im Schwarzwaldkristallin schulterartige Verflachungen beobachtet werden, die in ähnlicher Gestalt um das Murgtal (E des Wehratales) auftreten. Ein geomorphogenetischer Zusammenhang mit den trogschulterähnlichen Verflachungen im Vorderen Wehratal ist nicht ausgeschlossen.

(2) Die Verflachungen um das Vordere Wehratal können nicht mit den Geste ins g r e n z en oder mit tektonisch begrenzten Gesteinsarealen begründet werden. Die trogschulterartigen Verflachungen schneiden die Gesteinsgrenzen. Das Eis hat die Gesteine offensichtlich gekappt.

(3) Der Sedimentcharakter des Profils Le 0316 weist auf einen sedimentdynamisch beruhigten Bereich hin, der auf den trogschulterartigen Verflachungen um das Tal eher zu erwarten ist als in dem noch eiserfüllten engeren Taleinschnitt.

\section{Danksagung}

Meinem Kollegen Professor Dr. G. STÄBLEIN vom Geomorphologischen Laboratorium der FU Berlin danke ich für briefliche und ausführliche mündliche Diskussionen über die geomorphogenetische Problematik. Für Diskussion am Aufschluß sei auch Herrn Dozenten D. H. SLUPETZKY vom Institut für Geographie der Universität Salzburg gedankt, ebenso den anderen Teilnehmern der Exkursion der Schweizerischen Geomorphologischen Gesellschaft auf das Blatt Wehr. - Den Bürgermeistern bzw. Baudezernenten der Gemeinden Hasel und Wehr danke ich dafür, daß ich fortwährend über neue Aufschlüsse im Bereich des Wehra- und Haseltales orientiert wurde. Nur durch die über die Kartierungszeit hinaus fortgesetzten Aufschlußaufnahmen konnte sich ein umfassendes Bild über die pleistozäne Gebietsentwicklung gemacht werden.

\section{Schriftenverzeichnis}

BARSCH, D. (1976): Das GMK-Schwerpunktprogramm der DFG: Geomorphologische Detailkartierung in der Bundesrepublik. - Z. Geomorph., N. F., 20: 488-498; Berlin, Stuttgart.

Dreimanis, A. (1979): The problems of waterlain tills. - In: Moraines and Varves, ed. by Ch. SCHLÜCHTER: 167-177; Rotterdam (Balkema). 
ERDMANNSDÖRFFER, O. H. (1903): Geologische und petrographische Untersuchungen im Wehrathal. — Mitt. Bad. Geol. Landesanst., 4: 145-195, Heidelberg.

HantKe, R. (1978): Eiszeitalter. Band 1: Die jüngste Erdgeschichte der Schweiz und ihre Nachbargebiete. Klima, Flora, Fauna, Mensch, Alt- und Mittel-Pleistozän, Vogesen, Schwarzwald, Schwäbische Alb, Adelegg. - 468 S., Thun (Ott).

JoAChim, H. \& Villinger, E. (1975): Erläuterungen zur Hydrogeologischen Karte von BadenWürttemberg 1:50 000. Markgräfler Land - Weitenauer Vorberge - Wiesental - Dinkelberg Hochrheintal - Wehratal (Bad Bellingen - Lörrach - Schopfheim - Säckingen). - 1-71; Freiburg i. Breisgau (Selbstverlag Geol. L.-Amt Baden-Württemberg).

Leser, H. (1976): Das GMK-Projekt. Bericht über die Arbeiten an geomorphologischen Karten der BRD. - Kartogr. Nachr., 26: 169-177; Bonn-Bad Godesberg.

- (1979a): Sedimente der Würm-Vereisung im Wehratal bei Todtmoos-Au im Südschwarzwald. - Ber. Naturf. Ges. Freiburg i. Br., 69: 31-45; Freiburg i. Breisgau.

- (1979b): Geomorphologische Karte der Bundesrepublik Deutschland 1:25 000, Blatt 4, 8313 Wehr. - Berlin (in Kommission bei GeoCenter, Stuttgart).

- (1980): Zum Problem rißzeitlicher Sedimente im Wehra-Tal (Südschwarzwald). - Oberrhein. Geol. Abh., 29: 59-69; Karlsruhe.

- \& StÄblein, G. (1978): Legende der Geomorphologischen Karte 1:25 000 (GMK 25). 3. Fassung im GMK-Schwerpunktgrogramm. - Berliner Geogr. Abh., 30: 79-90; Berlin (Selbstverlag Inst. f. Phys. Geogr. FU Berlin).

Pfannenstiel, M. (1969): Grundmoräne des Riß-eiszeitlichen Wehragletschers bei Öflingen. Ber. Naturf. Ges. Freiburg i. Br., 59: 31-34; Freiburg i. Breisgau.

- \& Raнm, G. (1964): Die Vergletscherung des Wehratales und der Wiesetäler während der Rißeiszeit. - Ber. Naturf. Ges. Freiburg i. Br., 54: 209-278; Freiburg i. Breisgau.

RAнм, G. (1961): Über den Betrag des Wehratal-Abbruches. - Ber. Naturf. Ges. Freiburg i. Br., 51: 273-275; Freiburg i. Breisgau.

Scheffer, F. \& Schachtschabel, P. (1976): Lehrbuch der Bodenkunde. - 9. Auflage, 394 S., Stuttgart (Enke).

Schwarzbach, M. (1978): Glazigene Sichelmarken als Klimazeugen. - Eiszeitalter u. Gegenwart, 28: 109-118; Öhringen/Württ.

Shaw, J. \& Archer J. (1979): Deglaciation and glaciolacustrine sedimentation conditions, Okanagan Valley, British Collumbia, Canada. - In: Moraines and Varves, ed. by Ch. Schlüchter: 347-355; Rotterdam (Balkema).

StÄBleIN, G. (Ed.) (1978): Geomorphologische Detailaufnahme. - Beiträge zum GMK-Schwerpunktprogramm I. Berliner Geogr. Abh., 30, 90 S.; Berlin (Selbstverlag Inst. f. Phys. Geogr. FU Berlin). 